\title{
Quelques spécificités de la modalisation dans le discours scientifique
}

\section{Claude Sionis}

\section{(2) OpenEdition}

1 Journals

\section{Édition électronique}

URL : http://journals.openedition.org/asp/1602

DOI : 10.4000/asp. 1602

ISBN : 978-2-8218-0388-6

ISSN : 2108-6354

\section{Éditeur}

Groupe d'étude et de recherche en anglais de spécialité

Édition imprimée

Date de publication : 1 décembre 2002

Pagination : 49-59

ISSN : 1246-8185

\section{Référence électronique}

Claude Sionis, «Quelques spécificités de la modalisation dans le discours scientifique », ASp [En ligne], 35-36 | 2002, mis en ligne le 12 août 2010, consulté le 19 avril 2019. URL : http://

journals.openedition.org/asp/1602; DOI : 10.4000/asp.1602

Ce document a été généré automatiquement le 19 avril 2019.

Tous droits réservés 


\title{
Quelques spécificités de la modalisation dans le discours scientifique
}

\author{
Claude Sionis
}

\section{Introduction}

$1 \quad$ La $^{1}$ modalité dans le discours est sans doute l'un des domaines dont la maîtrise est la plus nécessaire pour des locuteurs-rédacteurs scientifiques, elle est aussi l'un des domaines les plus problématiques et exige qu'une sorte d'état des lieux soit fait avant d'opter pour une stratégie d'analyse définie. C'est cet état des lieux préliminaire que le présent article se propose de faire.

2 Les premiers chercheurs en anglais scientifique comme Barber (1962) se sont essentiellement préoccupés du rôle joué par les verbes modaux dans le contexte de la modalisation. Barber recense $16 \%$ de verbes au mode fini accompagnés de modaux sur un corpus de 23400 mots, cette prise en compte des seuls verbes modaux se retrouve chez les «rhétoriciens» du discours scientifique comme Trimble (1985) qui décrit la modalité comme étant un «semi-jargon » et qui ne justifie l'étude de should, must, may et can que dans le genre rédactionnel « instructions ». Meyer (1998), éminent représentant de l'école allemande d'anglais de spécialité dont le présent article s'inspire pour de nombreux points, adopte la même attitude vis-à-vis de la modalité dans le discours scientifique et ne considère guère que le rôle joué par les verbes modaux. Les représentants de l'école "générique» en LSP, Swales (1990) et Bhatia (1993), ont ajouté une dimension sémantique à la notion de modalité. Les sociologues de la science (Bazerman 1988; Forstop 1989 Lynch \& Woolgar 1990) ont présenté la démarche de modalisation comme étant absolument consubstantielle à la démarche scientifique, au point de transcender non seulement la notion de "genre » (rapport, note de recherche, exposé oral, etc.) mais même celle de « registre » (médecine, littérature, droit, etc.) 
3 La perspective d'étude de la modalisation dans le discours scientifique gagnerait sans doute à être élargie et à faire une place, non seulement au rôle joué traditionnellement par les verbes modaux, mais à d'autres éléments qui prendraient en compte, entre autres, les aspects cognitifs, pragmatiques, sémantiques et sociologiques du discours de la science $^{2}$.

\section{Modalisation et mondes possibles}

4 Il est courant d'opposer «langue générale » et "langue spécialisée », « communication quotidienne » et "communication à visée spéciale», ou "professionnelle», ou encore "scientifique", "technique», etc. Le scientifique, ou tout professionnel dont la communication dans l'exercice de sa profession est suffisamment typée pour se démarquer de la langue de Monsieur Tout le Monde, vivrait donc dans deux mondes dans lesquels communication "générale » et "spécialisée » seraient relativement distinctes (Sionis 2001). Cette notion de «double monde» doit cependant être comprise dans d'autres sens lorsque l'on traite de la modalité dans le discours scientifique.

5 L'auteur scientifique qui a recours à des procédés de modalisation de son discours (essentiellement à l'écrit pour ce qui suit) le fait fondamentalement pour signaler que les chances de réalisation d'un fait ou d'une hypothèse scientifiques ne sont ni assurées dans l'absolu, ni garanties par lui-même au moment où il écrit (Perkins 1983). Les raisons du recours à la modalisation sont certes plus nombreuses et plus subtiles, mais en se situant dans une perspective de réalisation ou de non-réalisation d'un fait, l'auteur scientifique désigne bel et bien deux mondes possibles : celui d'un monde « réel », dans lequel les faits et les hypothèses relèvent d'une réalité avérée et jusque-là immuable, et celui d'un monde « supposé ", espace propice à toutes les remises en question, tous les ajustements et toutes les révolutions.

6 Les notions de "réel » et de "supposé » ne relèvent certes pas des mêmes catégories logiques. Selon von Wright (1951), « réel» relèverait du domaine de l'ontologique alors que « supposé » relèverait du domaine de l'épistémique. Néanmoins, le cadre de référence de la science, et en particulier de la représentation écrite de celle-ci, avec le positionnement énonciatif de nature essentiellement rhétorique du scripteur qu'elle suppose, rend commode l'opposition réel/supposé quand elle se limite au strict domaine de l'épistémique.

7 Une autre vision voisine serait celle d'un monde "réel » où les théories et les faits seraient acceptés ou rejetés en fonction de critères, par exemple, fonctionnels, mais ne souffrant aucune réserve en dehors de cette alternative binaire, et un autre «monde irréel» dans lequel vérité et fausseté fluctueraient au gré de conditions subjectives, aléatoires, incontrôlées, etc. Ce «monde irréel » fournirait un espace discursif mouvant dans lequel viendraient s'insérer la discussion, la prise de recul, le doute rhétorique qui permet d'interroger des concepts jusque-là inébranlables, d'établir des rapports dialectiques entre les idées, etc.

8 Au-delà de l'étude des procédés syntaxiques ou discursifs qui caractérisent la modalisation dans une langue naturelle donnée (voir plus bas), il semblerait que le discours scientifique soit caractérisé par certaines nécessités de modalisation qui obéissent à des stratégies sociolinguistiques: être accepté et approuvé par une communauté de recherche, appuyer sa propre recherche, éventuellement sujette à 
caution, sur la recherche d'autrui, antérieure et non discutée. Les chercheurs du domaine des langues de spécialité ou de la sociologie des sciences qui se sont penchés sur cet aspect de la modalisation sont nombreux (Swales 1971, 1981, 1990; Gilbert \& Mulkay 1984 ; Myers 1985 ; Mulkay 1991).

Pour ces auteurs, il y aurait des justifications internes spécifiques à la démarche de (re)présentation du savoir scientifique pour l'usage de procédés de modalisation linguistiques ou discursifs. La différenciation se justifie si l'on considère, par exemple, le recours à des choix de type lexical (tel verbe modal, ou tel adverbe, plutôt que tel autre) mais aussi à des constructions spécifiques dans lesquelles l'organisation du discours (par ex. the process is not always what people expect) relève d'un type de hedges (voir Marchais 2002, pour le phénomène de "surmodalisation »). Ces procédés refléteraient des attitudes idéologiques ou des intentions de communication qui seraient des caractéristiques inhérentes à la démarche scientifique et à sa représentation écrite.

10 À cet égard, l'usage des temps grammaticaux peut prêter à des interprétations ambiguës : la science qui parle d'elle-même au présent se réfère obligatoirement à un état du monde qui pourrait bien être autre que ce que l'on imagine au moment présent. Si l'on conçoit et présente un état du monde différent au moment où l'on formule des hypothèses, on le conçoit comme pouvant être vrai ou réel dans un monde non conforme au monde actuel. L'autre situation est de concevoir un état des choses différent dans le monde réel, mais à un autre moment de ce monde réel.

11 Une caractéristique possible de la modalité est la relativisation de la valeur de vérité d'une phrase par rapport à un ensemble de mondes possibles. Parler de mondes possibles c'est parler des façons dont les gens peuvent concevoir le monde comme différent. On est au cœur de la démarche scientifique et de la notion de "progrès scientifique ».

\section{La verbalisation de l'évaluation}

Dans la démarche scientifique, il y a cependant au moins deux grandes catégories de jugements évaluatifs qui se traduisent par des actes de parole différents.

L'évaluation peut être factive : «c'est juste », « c'est correct », « c'est faux », « c'est bon », etc. Ou elle peut être interprétative : «je pense que», "je déduis que», «je conclue que ", etc. La modalisation sous sa forme stratégique et sociolinguistique va surtout s'appliquer à la première catégorie, l'évaluation factive, dans la mesure où un jugement personnel du chercheur va aboutir à une prise de position relativement à d'autres prises de position ou théories d'autres chercheurs (notion d'acceptabilité sociale et stratégique).

La modalisation sous sa forme introspective et psycholinguistique va surtout s'adresser à la seconde catégorie, l'évaluation interprétative, où la relativisation du sens d'une phrase va se justifier par rapport à des jugements antérieurs du même chercheur ou en fonction d'un raisonnement du type « questions rhétoriques » ou auto-adressées.

La frontière entre les deux sortes d'évaluation n'est cependant pas aussi nette que l'on pourrait croire d'emblée : un énoncé du type "c'est correct-faux-bon », etc. est toujours produit par un locuteur-rédacteur dont la position sociale et scientifique, la tâche ou le propos, font l'objet d'une description aussi minutieuse et complète que l'exige les genres communicationnels formellement modalisés et contraints de la recherche. L'article de haut niveau, vecteur par excellence du savoir scientifique, en est l'exemple canonique. 
Dès lors, une part d'interprétation subjective, et donc interprétative, est toujours présente dans l'énoncé factif de tel ou tel auteur.

Les verbes dits «illocutionnaires » qui caractérisent les actes de parole ne référant qu'à un acte et non à la relativisation de la validité d'un état du monde - dans un énoncé du type "J'affirme que tous les hommes sont mortels", dire que quelque chose est compatible avec ce que j'affirme n'a pas de sens. La vérité de l'assertion ne dépend pas de mon opinion et ne se situe pas sur une échelle d'acceptation variable de mon énoncé. L'illocution serait donc étrangère à la notion de modalité (Kiefer 1987 : 87).

\section{Dictum et modus}

En ce qui concerne le discours scientifique et l'aide à la rédaction en langue étrangère que l'on pourrait apporter dans le domaine de la modalité à un rédacteur avancé, il peut s'avérer utile d'envisager un autre ensemble de critères. Selon Bally (1942), la modalité est présente dans toute évaluation de la part du locuteur. Pour Bally, chaque énoncé comporte deux composantes : ce qui est écrit ou dit (le «dictum»), et la façon dont cela est écrit ou dit (le «modus »). Dans un énoncé comme "Je pense que c'est faux », «Il est probable que c'est faux ", "Cela doit être faux » le dictum est : " c'est faux », tout le reste relève de la modalité (le modus). La modalité serait donc définie comme l'attitude cognitive, émotive, ou volitive du locuteur envers un état du monde. Une mise en garde s'impose à ce niveau : les attitudes doivent être distinguées des états ou d'une certaine description du monde fournie par le locuteur-rédacteur. L'expression linguistique de telles descriptions ne relève pas de la modalité. Dans certains cas, l'attitude et la cause se manifestent par deux constructions syntaxiques différentes :
1. Je m'étonne qu'on ne me réponde pas (je m'étonne est remplaçable par des expressions du type " je juge étonnant », « il est surprenant », etc.)
2. Je m'étonne de ce qu'on ne me répond pas (la construction en « de " = «le fait que ", exclut une substitution par des expressions modalisantes comme en (1) et décrit je m'étonne comme un résultat et de ce qu'on ne me répond pas comme une cause.

Le prédicat «s'étonner » ne relève de la modalité que dans le premier cas (Kiefer 1998 : 592).

Dans une perspective d'aide à la rédaction scientifique, ce sont les différentes façons d'exprimer le modus qui devraient être mises à la disposition de l'apprenant-rédacteur. Pour des raisons de facilité didactique et de programmation informatique, il est probable qu'un regroupement des différentes formulations possibles devrait se faire en fonction des catégories traditionnelles d'expression de la modalité (tableau 1). 
Tableau 1. Catégories traditionnelles d'expression de la modalité

\begin{tabular}{|l|l|}
$\begin{array}{l}\text { Verbe d'opinion : I think the reasoning } \\
\text { is right }\end{array}$ & $\begin{array}{l}\text { Le dictum est « it is right », tout le reste relève de } \\
\text { la modalité. }\end{array}$ \\
Adverbe : It is probably right \\
$\begin{array}{l}\text { Verbe modal : It needs/has to....be right } \\
\text { Auxiliaire modal : It must be right } \\
\text { Verbe de volition : I want it to be right }\end{array}$ \\
\begin{tabular}{l}
... \\
\hline
\end{tabular}
\end{tabular}

Il s'agirait donc d'identifier l'attitude cognitive, émotive ou volitive du rédacteur vis-à-vis d'un état des choses, en l'occurrence la proposition qu'il souhaite modaliser. L'on traiterait de l'attitude vis-à-vis de l'état des choses et non de l'état des choses lui-même. La phrase peut donc être scindée en deux parties en fonction du prédicat qui indique le modus et celui qui indique le dictum.

\begin{tabular}{|l|l|}
\hline $\begin{array}{l}\text { On pourrait s'étonner } \\
\text { We could wonder } \\
\text { MODUS }\end{array}$ & $\begin{array}{l}\text { que le phénomène se produise quand.... } \\
\text { that the phenomenon occurs when } \\
\text { DICTUM }\end{array}$ \\
\hline $\begin{array}{l}\text { Il est étonnant } \\
\text { It is surprising } \\
\text { DICTUM }\end{array}$ & $\begin{array}{l}\text { que le phénomène puisse se produire quand... } \\
\text { that the phenomenon can occur when... } \\
\text { MODUS }\end{array}$ \\
\hline
\end{tabular}

On peut argumenter sur le fait que $A$ relève d'une relation attitude-état du monde et $B$ d'une relation cause-effet (la deuxième partie de la phrase $B$ exprimant le modus agirait comme cause et la première serait l'effet). La présence du modal can en B déplace la modalisation vers la deuxième proposition, ce qui pose la question de la hiérarchie des marqueurs de modalisation: peut-on parler d'autre part de "force modalisatrice", comme l'on parle de «force illocutionnaire »?

21 Une autre interprétation de B serait de considérer les deux parties de la phrase comme deux modalités différentes : la première étant un modus de dicto, la seconde un dictum + un modus de re. Pour faciliter la discrimination entre dictum et modus, il conviendrait peut-être d'utiliser la distinction que fait Frege entre pensée, jugement et assertion. Si l'apprenant devait identifier les différente parties de phrase en fonction de ces appellations, il attribuerait la pensée au dictum (la formulation non modalisée ou le contenu propositionnel) et le jugement au modus (qui reflète l'attitude du rédacteur face aux choses), l'assertion prise dans un sens très large serait alors l'ensemble dictum-modus.

Dans le cas qui nous préoccupe, la modalité, il faudrait surtout déterminer où se place le modus et sans doute établir des taxinomies de prédicats. Par exemple, si l'on peut dire qu'un verbe d'attitude, souvent de type épistémique (je crois, j'estime, je m'étonne, j'admets, etc.) non causatif, transitif, et construit de manière personnelle, se trouve dans la proposition, c'est cette proposition qui porte le modus. La proposition enchâssée avec QUE/THAT est généralement le dictum (voir néanmoins plus haut le contre-exemple B). 
23 Si l'on a affaire à une seule proposition, il est plus difficile de séparer dictum et modus. Le modus est alors exprimé sous diverses formes :

Adverbe : Le phénomène va probablement se produire.

Verbe modal : Le phénomène doit se produire.

Une marque sur le verbe (temps, aspect) : Le phénomène ne se serait jamais produit (plus complexe).

\section{Modalité de phrase et modalité verbale}

La première est une catégorie sémantique verbalisable sous différentes formes morphosyntaxiques, la seconde est une catégorie morphosyntaxique. La modalité de phrase est la valeur modale de certains types de phrases. Plusieurs types de phrases peuvent exprimer la même modalité de phrase.

Tableau 2. Exemple

\begin{tabular}{|l|l|}
\hline Modalité de phrase & Types de phrases en anglais \\
\hline \hline Interrogativité & $\begin{array}{l}\text { Questions yes-no } \\
\text { Questions disjointes (x or y ?) } \\
\text { Wh-questions (Where? What? Who?...) }\end{array}$ \\
\hline
\end{tabular}

Le sens d'une modalité de phrase reflète l'attitude communicationnelle du locuteurrédacteur vis-à-vis de son interlocuteur-lecteur et vis-à-vis de l'objet de la communication.

Tableau 3. Exemples

\begin{tabular}{|l|l|}
\hline Types de phrases & Formulations possibles de l'attitude du locuteur-rédacteur \\
\hline Déclaratives & $\begin{array}{l}\text { The speaker takes for granted that... } \\
\text { The writer considers that... } \\
\text { This author regards phenomenon X as being... }\end{array}$ \\
\hline Interrogatives & $\begin{array}{l}\text { The authors wonder about... } \\
\text { The problem is a matter of X or Y } \\
\text { Now the issue is to know what... } \\
\text { Whether } \mathrm{X} \text { is true is for us to determine }\end{array}$ \\
\hline Exclamatives & $\begin{array}{l}\mathrm{X} \text { is astonished about the fact that } \\
\text { One may find the fact surprising } \\
\text { This is all the more surprising as... }\end{array}$ \\
\hline
\end{tabular}




\begin{tabular}{|l|l|}
\hline Impératives & $\begin{array}{l}\text { We now require you to... } \\
\text { It is then mandatory to } \\
\text { Consider } \mathrm{X} \text { and assess the validity of } \mathrm{Y} \text { in these circumstances }\end{array}$ \\
\hline Optatives & $\begin{array}{l}\text { X wants ... to happen } \\
\text { We wish ... to be the case } \\
\text { X's desires are... }\end{array}$ \\
\hline
\end{tabular}

L'intégration d'une telle base de données à un logiciel d'enseignement à distance semble tout à fait réalisable; le problème posé par cette approche est cependant la difficulté de proposer des formulations non ambiguës et clairement liées à tel ou tel type de phrase. Les exclamatives et les déclaratives sont sémantiquement et syntaxiquement très proches, de même que les optatives et les impératives. Par exemple, une formulation très fréquente en science comme :

Either $\mathrm{X}$ happens or the process is no longer valid

Optative ? Impérative ? Ou Interrogative?

L'attitude exclamative contient l'attitude déclarative puisque s'étonner d'une situation implique que cette situation est reconnue et intégrée. Les déclaratives ne devraient-elles pas être considérées comme les seuls types de phrases non modalisables dans la mesure où les autres types de phrases ne sont modalisantes que par rapport aux déclaratives? Les déclaratives ne relèveraient alors que du dictum.

Une autre appréhension de la question serait de considérer que modalités épistémiques et assertives (et interrogatives) sont sur un même plan, de sorte qu'une modalité épistémique peut ne pas pouvoir modaliser une déclarative. Ainsi :

He may be tired.

Is he tired?

*May he be tired?

Il est impossible de cumuler deux modalités de même niveau.

\section{Modalité épistémique et modalité déontique}

La modalité épistémique concerne le savoir et la croyance, la modalité déontique renvoie à la nécessité ou la possibilité d'accomplir une action et introduit la notion d'autorité, de pouvoir, de licence, etc. La réalisation linguistique de ces types de modalité présente cependant de nombreuses ambiguïtés.

Si l'on prend un exemple rendu typiquement ambigu par l'usage de may:

He may think that...-> un premier sens est perhaps he thinks that... (modalité épistémique)

He may think that...->un deuxième sens est he is permitted to think that... (modalité déontique)

31 L'ambiguïté de may sera une difficulté dans la perspective didactique d'un support EAD, mais He may know the answer peut seulement être épistémique. L'interprétation dépend du contexte. En science, on conçoit mal que l'accès à une réponse ou à une vérité puisse être 
conditionnel ou dépendre d'une autorisation ou d'un droit, mais on peut argumenter sur ce point. L'ambiguïté du sens des modaux est illustrée par les exemples suivants :

Following these observations the conclusion must be that...

Obligation "contingente» découlant des circonstances du type: si $\mathrm{X} \rightarrow \mathrm{Y}$ Donc épistémique ? OU Obligation absolue, purement déontique?

L'usage non-épistémique de must peut exprimer le fait que l'environnement, les circonstances, sont un empêchement.

Researchers cannot recognise the fact as...

Impossibilité épistémique, par exemple leurs connaissances excluent que...? OU Impossibilité déontique : ils n'ont pas le droit de ...on leur interdit de... (non fondé en science).

L'usage épistémique de can en science indique qu'en fonction de ce que l'on sait, il n'est pas exclu que la proposition en question soit vraie. Lyons (1977) établit une différence entre "nécessité épistémique» et "possibilité épistémique»; ces deux types de modalités sont exprimés par des propositions différentes, en tout cas dans la majorité des usages de langue générale. Une proposition est épistémiquement nécessaire si elle est logiquement impliquée (entailed) par ce que l'on sait; une proposition est épistémiquement possible si elle est compatible avec ce que l'on sait.

Dans le cas du discours scientifique qui nous intéresse, possibilité ou nécessité ne se distinguent pas toujours aisément :

Sulphur and oxygen must be mixed to produce sulphur dioxide (nécessité et possibilité)

Cet aspect est cependant important en science, comme Lyons (1977: 845) le signale en quelque sorte «par défaut »:

epistemic possibility [...][derives from] the child's acquisition of his native language, from uncertainty and doubt [...] the objectification of epistemic necessity and possibility is a rather sophisticated and impersonal process which plays little part in ordinary non-scientific discourse; and languages [...] are semiotic systems that are used primarily for non-scientific discourse.

\section{Modalité et actes de parole} déontique » et la " possibilité déontique » sont des notions sémantiques, mais l'obligation ou la permission appartiennent à la pragmatique. Imposer une obligation à quelqu'un ou accorder une permission sont des actes de parole. Quand un chercheur écrit : We can quote $\mathrm{X}$ in this context, il énonce une possibilité déontique, mais il s'autorise aussi à le faire. Toutefois, ce n'est un acte de parole «heureux» que si le rédacteur a l'autorité ou la légitimité pour s'accorder cette permission. Cette légitimité $a$, on l'espère, été établie et argumentée par le texte-avant. C'est tout le processus de l'argumentation scientifique qui se justifie s'il aboutit à un acte de parole reconnu comme tel par le lecteur.

Dans la langue d'usage général, l'arrière-plan déontique est plus facile à garantir, par exemple dans une conversation entre professeur et élève où les places sociales de l'un et 
de l'autre sont posées explicitement et souvent renforcées par le cadre physique et psychologique (cf. modèle « d'événement de parole » s-p-e-a-k-i-n-g de Hymes,1972).

\section{Modalité objective et modalité subjective}

eut-être vaudrait-il mieux parler de modalité objective, celle qui se réfère à la réalité, à la description du monde, et, malgré la prétendue objectivité du discours de la science, de modalité subjective, celle qui est l'expression des sentiments et des croyances du locuteur - les deux modalités étant d'ailleurs épistémiques. Comme le remarque Kiefer (1998: 594),

[...] les différences sémantiques entre modalité épistémique subjective et objective sont importantes, les phrases modalisées objectivement sont des affirmations de faits et comme telles peuvent être niées ou remises en question, elles peuvent se produire dans des propositions en « $\mathrm{Si}$ » et elles peuvent être enchâssées dans des prédicats factifs. À l'opposé, les phrases épistémiques subjectives expriment les croyances du rédacteur et ne sont pas des affirmations de faits.

Cette distinction est capitale pour le discours scientifique car, dans ce dernier cas, il est impossible de les nier ou de les remettre en question. C'est bien ce qui se passe dans la réalité du débat scientifique où seules les propositions épistémiquement objectives sont passibles de contestation sur les faits (par ex. en leur opposant d'autres faits avérés par d'autres procédés) car elles seules peuvent être confrontées à la "réalité objective " (littéralement: «à laquelle on peut objecter»), une collocation qui exprime bien sa fonction en science.

Cet aspect est souligné par Lyons (1977 : 805) également pour la langue générale :

[...] few linguists have even considered the possibility that epistemic modality could be anything other that a matter of the speaker's attitude towards the propositional content of his utterance, and most discussions of mood and modality in linguistics seem to take it for granted that epistemic modality is subjective in this sense.

Bien qu'applicable originellement à la langue générale, l'analyse de la modalité présentée par Leech et Svartvick (1983: 128-129) selon une «échelle de probabilité » pourrait être une amorce de réflexion sur les rapports entre possibilité et probabilité des concepts énoncés en science. L'échelle de probabilité entend se substituer à une relation binaire vrai-faux, ou pour reprendre le concept des deux mondes possibles évoqué plus haut, à une relation monde réel-monde supposé. Les deux extrémités de l'échelle sont impossibilité et certitude (ou nécessité logique). Les échelons intermédiaires sont possibilité, probabilité, improbabilité, etc. La démarche consisterait pour le pédagogue à associer des modes de réalisation linguistique à ces catégories sémantiques. Comme la liste des types de modalités linguistiques donnée plus bas le rappelle, les trois types de réalisations linguistiques sont les verbes modaux, les constructions «It + that ", et les adverbes:

You may be right.

It is possible that you are right.

Perhaps you are right.

Comme il a été signalé plus haut au sujet de la distinction modus-dictum pour l'attitude et la cause, le fait et la théorie se manifestent aussi par des constructions syntaxiques différentes. Au sujet de can, may, could et might, Leech et Svartvick (ibid, 128, cités par 
Meyer 1998) distinguent la possibilité du fait (modalité «factuelle’) de la possibilité de l'idée (modalité « théorique'):

Possibilité du fait: The railways may be improved = It is possible that the railways will be improved = perhaps $/$ maybe the railways will be improved . Possibilité de l'idée: the railways can be improved = it is possible for the railways to be improved.

La possibilité théorique (can) est " plus faible » que la possibilité factuelle (may). "The railways can be improved" dit seulement qu'en théorie, les chemins de fer sont améliorables, c'est-à-dire qu'ils ne sont pas parfaits. D'autre part, l'énoncé « The railways may be improved» pourrait suggérer qu'il existe des plans précis pour l'amélioration. Selon ces auteurs, can aurait finalement le même sens que sometimes dans des énoncés traduisant la possibilité en général :

Lightning can be dangerous = lightning is sometimes dangerous .

Dans un contexte de discours scientifique, cette distinction est moins évidente. Un énoncé comme :

Ethanol can be mixed with the X-Y compound.

Insiste essentiellement sur la possibilité physique (par exemple, «sans résultats désastreux ») du mélange éthanol-composé X-Y.

Ethanol is sometimes mixed with the $\mathrm{X}-\mathrm{Y}$ compound.

Insiste pour sa part, selon nous, sur l'occurrence éventuelle de ce mélange (signifiant par exemple: "une option possible ouverte au chercheur est de mélanger l'éthanol et le composé $\mathrm{X}-\mathrm{Y} »)$. Cette distinction faite par Leech et Svartvick entre possibilité du fait et possibilité de l'idée est cependant utile pour les différences de sens et d'emploi des verbes modaux, un aspect de la modalité en science qui devrait être traité prioritairement dans l'optique de faire acquérir au rédacteur-apprenant une aptitude de base dans ce domaine. Elle est aussi utile pour faire prendre conscience à ce même apprenant qu'il existe une gradabilité sémantique et linguistique de la modalité. À cet égard, l'établissement d'une taxinomie des types de modalités réalisées linguistiquement par le discours scientifique nous semble indispensable dans toute démarche d'aide à distance à la rédaction scientifique. Une amorce de recensement de ces types pourrait inclure les formulations évoquées plus haut.

Tableau 4. Types de modalité réalisées linguistiquement par le discours scientifique

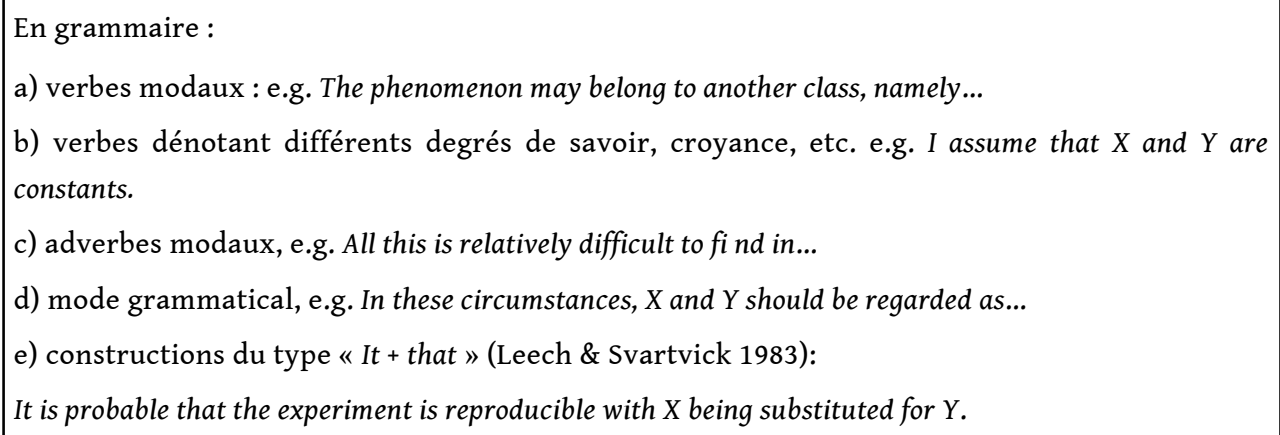


En sémantique :

Les différentes façons pour un locuteur-rédacteur d'indiquer que ce qu'il dit ou écrit n'est pas un fait mais plutôt :

a) qu'il spécule l'existence du fait (au début d'une recherche, l'intuition est capitale)

b) qu'il présente le fait comme une déduction (la méthode d'analyse, n'est pas infaillible)

c) qu'il a lu ou entendu parler du fait (la référence à des lois, d'autres faits et des théories établis et reconnus. La modalisation objective par l'auteur équivaut à une remise en doute de ces éléments, démarche essentielle en science.)

d) qu'il faut faire confiance à l'expérimentation ou l'analyse et non à son intuition (et peut-être même à ses sens).

Dans ce domaine de la sémantique, les types a et $\mathrm{b}$ impliquent des jugements :

It might well find its root in...It can also be argued that...

Les types $\mathrm{c}$ et $\mathrm{d}$ impliquent des preuves (Attested, informed, reported evidence, etc.) Les prédicats choisis et les actes de parole appartiennent à des familles différentes. Il faut noter que dans les cas a, b et c, la modalité sera de type épistémique, mais que dans le cas $\mathrm{d}$, elle sera de type déontique.

L'auteur tient à remercier un évaluateur anonyme d'ASp dont les suggestions ont été bien volontiers intégrées au présent texte dans la mesure où elles étaient compatibles avec les positions qui y sont adoptées.

\section{BIBLIOGRAPHIE}

Bally, C. 1942. « Syntaxe de la modalité explicite ». Cahiers Saussure II, 3-13.

Barber, C.L. 1962. « Some measurable characteristics of modern scientific prose ». In Contributions to English Syntax and Phonology. Stockholm : Almquist \& Wiksell..

Bahtia, V. K. 1993. Analyzing Genre: Language Use In Professional Settings. Londres : Longman.

Bazerman, C. 1988. Shaping Written Knowledge. Madison : University of Wisconsin Press.

Biber, D. \& E. Finegan. 1988. « Adverbial stance types in English ». Discourse Processes 11, 1-34.

Biber, D. \& E. Finegan 1989. «Styles of stance in English: Lexical and grammatical marking of evidentiality and affect $»$. Text 9, 93-124.

Forstorp, P.-A. 1989. Discourse In Professional and Everyday Culture. Linkoping : University of Linköping.

Gilbert, G.N. \& M. Mulkay. 1984. Opening Pandora's Box: a Sociological Analysis of Scientific Discourse. Cambridge : Cambridge University Press. 
Hymes, D. 1972. « Models of the interaction of language and social life ». In Gumpertz J.J. \& D. Hymes (dir.), Directions in Sociolinguistics. the Ethnography of Communication. New York : Holt, Rhinehart and Winston.

Kiefer, F. 1987. « On Defining modality ». FoL XXI/1, 67-94

Kiefer, F. 1998. « Modality ». In Mey J. (dir), Concise Encyclopedia of Pragmatics. Londres : Pergamon.

Leech, G. \& J. Svartvick. 1983. A Communicative Grammar of English. Londres : Longman.

Levinson, S.C. 1983. Pragmatics. Cambridge : Cambridge University Press.

Lynch, M. \& S. Woolgar (dir.). 1990. Representation in Scientific Practice. Cambridge, MA : MIT Press.

Lyons, J. 1977. Semantics. Cambridge : Cambridge University Press.

Marchais, R. 2002. «La surmodalisation dans les articles de recherche scientifique anglophones : un phénomène culturel ? ». Mémoire de DEA en Sciences du langage, non publié disponible auprès de l'équipe ALPL, Université de Nantes, UFR Langues, BP 81227 - 44312 Nantes Cedex 3.

Meyer, H. J. 1989. « Modality in science texts ». Fachsprache 11/3-4, 127-135.

Mulkay, M. 1991. Sociology of Science. A Sociological Pilgrimage. Milton Keynes : Open University Press.

Myers, G. 1985. « Texts as knowledge claims. The social construction of two biology articles ». Social Studies of Science 15/4, 593-630.

Perkins, M. R. 1983. Modal Expressions in English. Londres : F. Pinter.

Salager-Meyer, F. 1994. « Hedges and textual communicative function in medical english written discourse ». English For Specific Purposes 13/2, 149-170.

Sionis, C. 2001. « Intermodal and inter-genre translation in scientific writing ». Fachsprache 23 /1-2, 41-51.

Swales, J. 1971. Writing Scientifi c English. Londres : Nelson.

Swales, J. 1981. Aspects of Article Introductions. Birmingham, UK : University of Aston.

Swales, J. 1990. Genre Analysis. English in Academic and Research Settings. Cambridge : Cambridge University Press.

Trimble, L. 1985. English for Science and Technology. A discourse approach. Cambridge : Cambridge University Press.

Von Wright, G.H. 1951. An Essay in Modal Logic. Amsterdam : North Holland.

\section{NOTES}

1. Version écrite de la communication faite au $23^{\mathrm{e}}$ Colloque du GERAS, 21-23 mars 2002, ENS LSH, Lyon

2. L'équipe de recherche nantaise $n^{\circ} 2323$, "Analyse linguistique et pratiques langagières ", s'est fixé comme objectif transversal d'envisager à terme la création d'un outil d'aide à la rédaction scientifique pour des chercheurs débutants et confirmés. Le travail de l'équipe se répartit entre une réflexion de type théorique et une mise en œuvre pratique de cet outil qui s'insérera dans une démarche plus générale d'enseignement universitaire à distance. 


\section{RÉSUMÉS}

L'article évoque les différents aspects de la modalité qui peuvent se rencontrer dans le discours scientifique écrit en anglais. Lorsqu'il représente par écrit son activité de recherche, le chercheur ressent le besoin d'employer différents procédés de modalisation pour exprimer des doutes, des précautions, des incertitudes, des possibilités limitées, des impossibilités complètes. Ce besoin, qui obéit à des contraintes psychologiques et sociologiques, se traduit par un choix de formes linguistiques et de stratégies rédactionnelles qui doivent concilier des exigences grammaticales et lexicales formelles et des « intentions de communiquer » conceptuelles. Les types traditionnels de modalisation (épistémique, déontique, objective et subjective) sont étudiés dans le cadre de l'apprentissage de l'écrit scientifique en anglais par des utilisateurs non-natifs de cette langue.

The article deals with various aspects of modality which can be encountered in written scientific discourse in English. When they represent their research activity in writing researchers feel the need to employ various processes of modalisation to express doubts, precautions, uncertainties, limited possibilities, complete impossibilities. This need, which obeys psychological and sociological constraints, is translated by a choice of linguistic forms and writing strategies which have to reconcile formal grammatical and lexical requirements and conceptual "communicative intents". The traditional types of modalisation - epistemic, deontic, objective, subjective - are studied within the framework of the learning of scientific writing in English by non-native users of this language.

\section{INDEX}

Keywords : learning, modality, scientific discourse, scientific writing

Mots-clés : apprentissage, discours scientifique, écrit scientifique, modalité

\section{AUTEUR}

\section{CLAUDE SIONIS}

Claude Sionis est Professeur à l'Université de La Rochelle et co-directeur de la Jeune Équipe « Analyse linguistique et pratiques langagières » (Université de Nantes). Il s'intéresse aux aspects pragmatiques de l'analyse du discours scientifique écrit et plus particulièrement aux stratégies de communication des chercheurs non-natifs. claude.sionis@wanadoo.fr 